\title{
Drug sales data analysis for outbreak detection of infectious diseases: a systematic literature review
}

Mathilde Pivette ${ }^{1,2,3^{*}}$, Judith E Mueller ${ }^{1,4}$, Pascal Crépey ${ }^{1,5}$ and Avner Bar-Hen²

\begin{abstract}
Background: This systematic literature review aimed to summarize evidence for the added value of drug sales data analysis for the surveillance of infectious diseases.

Methods: A search for relevant publications was conducted in Pubmed, Embase, Scopus, Cochrane Library, African Index Medicus and Lilacs databases. Retrieved studies were evaluated in terms of objectives, diseases studied, data sources, methodologies and performance for real-time surveillance. Most studies compared drug sales data to reference surveillance data using correlation measurements or indicators of outbreak detection performance (sensitivity, specificity, timeliness of the detection).
\end{abstract}

Results: We screened 3266 articles and included 27 in the review. Most studies focused on acute respiratory and gastroenteritis infections. Nineteen studies retrospectively compared drug sales data to reference clinical data, and significant correlations were observed in 17 of them. Four studies found that over-the-counter drug sales preceded clinical data in terms of incidence increase. Five studies developed and evaluated statistical algorithms for selecting drug groups to monitor specific diseases. Another three studies developed models to predict incidence increase from drug sales.

Conclusions: Drug sales data analyses appear to be a useful tool for surveillance of gastrointestinal and respiratory disease, and OTC drugs have the potential for early outbreak detection. Their utility remains to be investigated for other diseases, in particular those poorly surveyed.

Keywords: Disease outbreaks, Syndromic surveillance, Drug utilization, Nonprescription drugs, Communicable diseases, Epidemiology

\section{Background}

Since the mid-1990s and the raise of concerns about bioterrorism and emerging diseases, non-diagnosis-based data have increasingly been used for routine disease surveillance and outbreak detection [1]. The CDC defined "syndromic surveillance" as an investigational approach where health department staff, assisted by automated data acquisition and generation of statistical alerts, monitor disease indicators in real-time or near real-time to detect outbreaks of disease earlier than would otherwise be possible with traditional public health methods [2].

In such efforts, different registries have served as data sources for public health surveillance [1,3], including data on absenteeism at work or school [4], calls to health

\footnotetext{
* Correspondence: mathilde.pivette@ehesp.fr

${ }^{1}$ EHESP French School of Public Health, Sorbonne Paris Cité, Rennes, France

2Université Paris Descartes, MAP5, Paris, France

Full list of author information is available at the end of the article
}

helplines [5,6], emergency department consultations $[7,8]$, ambulance dispatching [9], or drug sales. Although unspecific, such data sources can have the advantage over diagnosis-based surveillance of providing information within short delays since the event and in readily available electronic form for relatively low-cost, while capturing large parts of the population.

Drug sales data analysis may overcome the limitation of poor specificity when groups of drugs are exclusively used for the disease or disease syndrome of interest. Furthermore, drug sales data may earlier capture changing population health status, as over-the-counter (OTC) sales and a dense network of pharmacies in most developed countries make drugs easily accessible to patients at the earliest appearance of their symptoms.

Despite this potential interest, no state of the art of drug sales based surveillance is available to date. The

C Biomed Central

(c) 2014 Pivette et al.; licensee BioMed Central Ltd. This is an Open Access article distributed under the terms of the Creative Commons Attribution License (http://creativecommons.org/licenses/by/2.0), which permits unrestricted use, distribution, and reproduction in any medium, provided the original work is properly credited. 
present systematic literature review therefore summarized the evidence for an added value of drug sales data for infectious disease surveillance. We limited the scope of the review to infectious diseases, as they represent a public health problem for which early and valid signal detection is of particular concern, in light of potentially rapid emergence and opportunity for control interventions.

\section{Methods}

We conducted a literature search from 1975 up to June 2012 to identify relevant peer-reviewed articles regarding surveillance of infectious diseases based on drug sales data. PRISMA guidelines were followed in the reporting of the review [10]. Published articles were searched for on electronic databases (Pubmed, Embase, Scopus, LILACS, African Index Medicus, Cochrane Library), using combinations of the following key words: ("surveillance" OR outbreak detection OR warning system) AND (overthe-counter OR "prescription drugs" OR pharmacy OR (pharmaceutical OR drug OR medication) sales). The search was limited to articles in English or French. There were no limitations on study settings.

To be included in the review, articles had to describe, test, or review an infectious disease surveillance based on drug sales data; and be original research that presented new data and results. We excluded studies that monitored chronic diseases, as well as prevalence studies whose purpose was not epidemic detection.

One reviewer screened and evaluated the titles and abstracts. Articles were widely included in a first stage. The full-text review and the final selection of the articles were made by two reviewers.

We reviewed and described the articles in terms of objectives, diseases studied, data sources, methodologies, and performance for real time surveillance. To describe methods and results, we separated the articles into three groups based on their main objective: descriptive retrospective studies, drug selection studies, and prediction studies. Outcomes selected to compare drug sales data to reference surveillance data of the corresponding disease were correlation measurements (strength and timeliness of the correlation) and indicators of outbreak detection performance (sensitivity, i.e. ability to identify true outbreaks; specificity, i.e. ability to identify true negative and timeliness of the detection).

\section{Results}

We screened a total of 3266 articles, of which 27 were included in the final review. The search and selection process is presented in Figure 1.

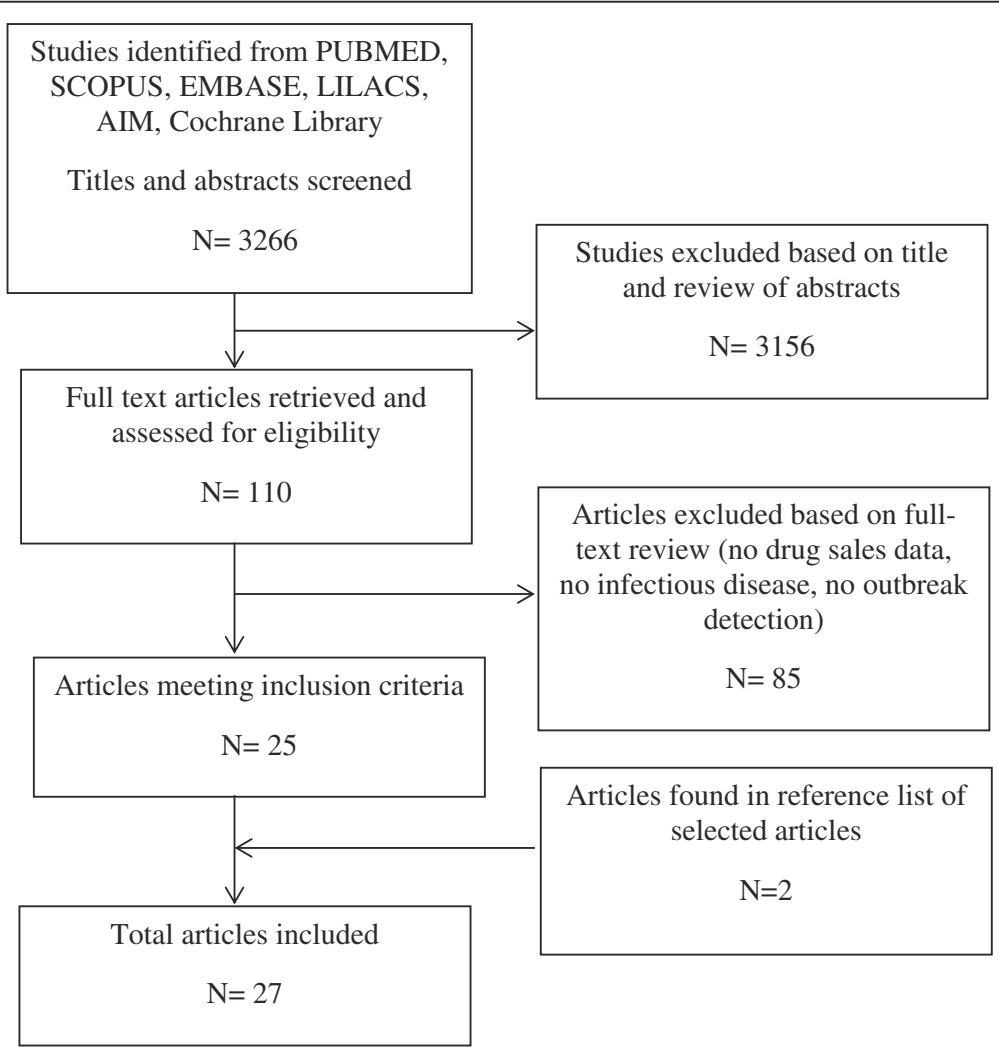

Figure 1 Flow chart of study selection process in a systematic review of drug sales data analysis for syndromic surveillance of infectious diseases. 


\section{Objectives of the studies}

Three types of studies were defined: retrospective descriptive studies, drug selection studies and prediction studies.

Nineteen of the 27 studies were descriptive retrospective studies assessing the strength of the correlation between drug sales and reference surveillance data of the corresponding disease or evaluating outbreak-detection performance [11-30]. Five studies used statistical algorithms to select groups of drugs that were closely associated with clinical surveillance data of a given disease and that would be most appropriate for future drugsales-based surveillance [21,31-34]. In a third group of three studies, the authors developed and evaluated statistical models to predict clinical surveillance data based on drug sales data [35-37]. Table 1 summarizes the studies in terms of their general characteristics.

\section{Diseases studied}

Most of the studies focused on respiratory illnesses (17 studies) [12,14,15,18,20-22,27-32,34-37] or gastrointestinal illnesses (11 studies) [14,16-19,23-26,32,33]. Only two other studies evaluated surveillance of pertussis [11] and syphilis [13].

\section{Populations surveyed}

Most of the studies were set in the United States $(n=16$ studies, $59 \%)$, followed by Canada $(n=4)$, France $(n=3)$, Japan $(n=3)$, the Netherlands $(n=1)$ and England $(n=1)$. Only one study was conducted in more than one country [25].

\section{Drug sales data sources}

In most retrospective studies, data were collected specifically for the purpose of the study from a sample of pharmacies [16,24-26] or from retailers [13,15,17,29]. For example, in a Canadian study [17], electronic data were provided by one major retailer for all of their 19 pharmacies in the study area.

Automatically compiled data sources were used in all the drug selection and prediction studies and in some retrospective studies. Drug sales data were routinely collected in samples of a city's or country's pharmacies. Such routine data collection systems were mainly implemented by research or public health groups, such as the Johns Hopkins Applied Physics Laboratory [20,21,35,36], the New York City Department of Health [14], the National Institute of Infectious Diseases in Japan [27], or the Real-Time Outbreak and Disease Surveillance Laboratory at the University of Pittsburg [19]. Data are available the day after the day's sales in those systems. In eight other studies, private marketing companies had automatically aggregated and made available drug sales data from a sample (2-90\%) of pharmacies in a given city or country [18,22,23,31-34,37].

\section{Methods and results by study types Retrospective studies}

Nineteen studies retrospectively compared drug sales data to gold standard reference data of the disease. Details are given in Table 2 .

Reference data of the disease included medical case reports [20-22], diagnostic registries of microbiological laboratories $[16,24,28,29]$, hospital admission or discharge data [18-22,27], or clinical emergency department data [14-16]. The selection of indicator drugs in these studies was based on the literature or expert opinion. For example, Edge et al. [17] selected all anti-nauseant and antidiarrheal OTC drugs for gastrointestinal surveillance. In Stirling et al. [26], pharmacists determined which common antidiarrheal drugs they would report.

Two methods were commonly used to compare drugsale and diagnostic data time series: correlation analysis and signal detection comparison (Table 2). Ten studies used cross-correlation function to measure the similarity of two curves and to determine the time lag at which the correlation between the datasets is maximized. Cross-correlation is a standard method to determine the time delay between two signals. In three studies, only correlation between the time series was examined without analyzing time-lagged relationship. Six studies used aberration detection methods to evaluate whether and by how long the date of signal detection by drug sales precedes the signal based on diagnostic data. The signal definition for aberration detection was based on either a simple threshold to define alerts [15] or more complex algorithms such as the Serfling method [14], ARIMA models [19], the simple moving average method (MA), the cumulative sum method (CUSUM) [11,16], or the exponentially weighted moving average (EWMA) [18]. These studies assessed the performance in terms of sensitivity, specificity and timeliness of disease outbreak detection. Five other studies [13,24-26,29] only evaluated whether drug sales showed a significant increase during a known epidemic period.

Twelve of 14 studies evaluating OTC sales retrospectively found significant correlations or a significant increase in drug sales [14-18,20-22,24-26,29]. Only two studies didn't found any consistent correlation. For example, Das et al. [14] found a poor correlation between OTC antidiarrheal drug sales and emergency department visits for diarrhea in New York City, with an $\mathrm{r}^{2}$ of 0.24 . They found however an increase in sales during a known outbreak of norovirus. OTC drug sales preceded clinical data in three of eight studies that analyzed timeline correlations [18,20,21]. For example, in Hogan et al. [18], the correlation coefficient between electrolyte sales 
Table 1 General characteristics of studies included in a literature review of drug sales data analyses for surveillance of infectious diseases

\begin{tabular}{|c|c|c|c|c|c|c|}
\hline Author & Study period & Location & Syndrome & Drug data sources & Drug status & Time scal \\
\hline \multicolumn{7}{|l|}{ RETROSPECTIVE STUDIES } \\
\hline \multirow[t]{2}{*}{ Sugawara et al. [27] 2012} & 2009/2010 & \multirow[t]{2}{*}{ Japan } & \multirow[t]{2}{*}{$\mathrm{ILI}$} & \multirow{2}{*}{$\begin{array}{l}\text { Prescription drug purchases from } \\
5275 \text { pharmacies }\end{array}$} & \multirow[t]{2}{*}{ Prescribed } & \multirow[t]{2}{*}{ Week } \\
\hline & 2010/2011 & & & & & \\
\hline Polgreen et al. [23] 2011 & Jan 1999- Sept 2007 & Ohio, USA & $\mathrm{Gl}$ & $\begin{array}{l}\text { IMS Health's Xponent database } \\
\text { (Data from retail pharmacies, } \\
\text { covering } 70 \% \text { of all prescriptions) }\end{array}$ & Prescribed & Month \\
\hline Chopra et al. [12] 2011 & April-Dec 2009 & Southeastern Michigan, USA & $\mathrm{ILI}$ & $\begin{array}{l}\text { In-site pharmacies of eight } \\
\text { hospitals in Southeastern } \\
\text { Michigan }\end{array}$ & Prescribed & Week \\
\hline Kirian et al. [19] 2010 & July 2003-Dec 2007 & San Francisco Bay area & $\mathrm{Gl}$ & $\begin{array}{l}\text { Sales available through the } \\
\text { National Retail Data Monitor in } \\
\text { three San Francisco Bay Area } \\
\text { counties (number of stores } \\
\text { reporting per week: 592-837) }\end{array}$ & OTC & Week \\
\hline Yoshida et al. [30] 2009 & Dec 2006-April 2007 & Sahai City, Osaka, Japan & $\mathrm{ILI}$ & $\begin{array}{l}\text { Questionnaires mailed to } 273 \\
\text { pharmacies. } 56 \% \text { responding } \\
\text { pharmacies. ( } 456,000 \text { people) }\end{array}$ & Prescribed & Week \\
\hline $\begin{array}{l}\text { Van den Wijngaard et al. } \\
\text { [28] } 2008\end{array}$ & $2001-2003$ & The Netherlands & $\mathrm{ILI}$ & $\begin{array}{l}\text { Foundation for pharmaceutical } \\
\text { statistics ( } 85 \% \text { of Dutch } \\
\text { pharmacies) ( } 13,8 \text { million people) }\end{array}$ & Prescribed & Week \\
\hline Edge et al. [17] 2006 & Jan 2001-April 2004 & Canada (one province) & $\mathrm{Gl}$ & $\begin{array}{l}\text { One major retailer with } 19 \\
\text { locations ( } 12 \% \text { of the pharmacies } \\
\text { in the region) }\end{array}$ & OTC & Week \\
\hline Das et al. [14] 2005 & Aug 2002-March 2005 & New York City & ILI and $\mathrm{Gl}$ & $\begin{array}{l}\text { New York City Department of } \\
\text { Health ( } 248 \text { NYC pharmacies, } \\
30 \% \text { of citywide sales) }\end{array}$ & OTC & Day \\
\hline Okhusa et al. [22] 2005 & Nov 2003-April 2004 & Japan & ILI & $\begin{array}{l}\text { Private marketing company ( } 1100 \\
\text { pharmacies, } 2 \% \text { of the } \\
\text { pharmacies in Japan) }\end{array}$ & OTC & Day \\
\hline Chen et al. [11] 2005 & March 2003-July 2004 & A rural county in New York State & Pertussis & $\begin{array}{l}\text { Medicaid database } \\
\text { reimbursement }\end{array}$ & Prescribed & Day \\
\hline Edge et al. [16] 2004 & $\begin{array}{l}\text { Jan-May } 2001 \\
\text { (Saskatchewan) March-June } \\
2000 \text { (Ontario) }\end{array}$ & $\begin{array}{l}\text { Battleford (Saskatchewan), } \\
\text { Walkerton(Ontario) }\end{array}$ & $\mathrm{Gl}$ & $\begin{array}{l}\text { One pharmacy in Battleford, one } \\
\text { pharmacy in Walkerton }\end{array}$ & OTC & Week \\
\hline Magruder et al. [21] 2004 & $2001-2003$ & Maryland-Washington-Virginia & $\mathrm{ILI}$ & $\begin{array}{l}\text { Johns Hopkins Applied Physics } \\
\text { laboratory ( } 300 \text { drugstores in the } \\
\text { Maryland-Washington-Virginia } \\
\text { area) }\end{array}$ & OTC & Day \\
\hline
\end{tabular}


Table 1 General characteristics of studies included in a literature review of drug sales data analyses for surveillance of infectious diseases (Continued)

\begin{tabular}{|c|c|c|c|c|c|c|}
\hline Couturier et al. [13] 2004 & $2001-2003$ & Paris and five french regions & Syphilis & $\begin{array}{l}\text { A centralized wholesaler } \\
\text { supplying all French private } \\
\text { pharmacies }\end{array}$ & Prescribed & Month \\
\hline Hogan et al. [18] 2003 & 1998-2001 & Pennsylvania, Indiana, Utah & ILI and Gl & $\begin{array}{l}\text { Information Resources, Inc., } \\
\text { private company ( } 90 \% \text { market } \\
\text { share in all the region) }\end{array}$ & OTC & Week \\
\hline Magruder [20] 2003 & $2001-2002$ & Maryland-Washington-Virginia & ILI & $\begin{array}{l}\text { Johns Hopkins APL ( } 300 \\
\text { drugstores in the Maryland- } \\
\text { Washington-Virginia area) }\end{array}$ & OTC & Day \\
\hline Davies et al. [15] 2003 & $\begin{array}{l}\text { 1998/1999, 1999/2000, 2000/ } \\
2001\end{array}$ & Nottingham city & ILI & $\begin{array}{l}\text { Retailers (Boots the chemist, 30\% } \\
\text { of market share) in the hospital } \\
\text { area, and a pharmaceutical } \\
\text { company (Reckitt Benckiser) at } \\
\text { national level }\end{array}$ & OTC & Week \\
\hline Stirling et al. [26] 2001 & Jan-May 2001 & North Battleford, Saskatchewan & Gl & Three pharmacies & OTC & Week \\
\hline Proctor et al. [24] 1998 & 1993 & Milwaukee (Wisconsin, USA) & Gl & One pharmacy & OTC & Day \\
\hline Rodman et al. [25] 1997 & $\begin{array}{l}\text { 1993(Milkauwee)/1994 (Las } \\
\text { Vegas)/1996 (Collingwood, } \\
\text { Kelowna, Cranbrook) }\end{array}$ & $\begin{array}{l}\text { Milwaukee (Wisconsin); } \\
\text { Collingwood (Ontario), Kelowna, } \\
\text { Cranbrook (British Colombia), Las } \\
\text { Vegas(Nevada) }\end{array}$ & $\mathrm{Gl}$ & $\begin{array}{l}\text { One pharmacy in Milwaukee, } 3 \\
\text { pharmacies in Collingwood, } 10 \\
\text { to } 12 \text { pharmacies in Kelowna and } \\
\text { Cranbrook. }\end{array}$ & OTC & Month \\
\hline Welliver et al. [29] 1979 & 1976/1977 & Los Angeles & ILI & One large supermarket chain & OTC & Week \\
\hline \multicolumn{7}{|l|}{ DRUG SELECTION STUDIES } \\
\hline Pelat et al. [33] 2010 & $2000-2009$ & France & Gl & $\begin{array}{l}\text { IMS Health ( } 7,500 \text { pharmacies in } \\
2000-13,300 \text { in } 2008,59 \% \text { of the } \\
\text { pharmacies in France, covering } \\
\text { all continental France) }\end{array}$ & OTC and prescribed & Week \\
\hline Cami et al. [31] 2009 & Jul 2003- Dec 2006 & $\begin{array}{l}\text { Region around the city of } \\
\text { Houston, TX, USA }\end{array}$ & ILI & $\begin{array}{l}\text { AC Nielsen Corp (pharmacy sales } \\
\text { in a region around Houston) }\end{array}$ & OTC & Week \\
\hline Wallstrom et al. [34] 2007 & $2002-2004$ & Pennsylvania, USA & ILI & $\begin{array}{l}\text { AC Nielsen Corp (pharmacy sales } \\
\text { in western Pennsylvania) }\end{array}$ & OTC & Week \\
\hline Li et al. [32] 2005 & Jan 1998- Dec 2001 & Pennsylvania, USA & ILI and Gl & $\begin{array}{l}\text { Information Resources, Inc., } \\
\text { private company ( } 90 \% \text { market } \\
\text { share in the region) }\end{array}$ & OTC & Week \\
\hline Magruder et al. [21] 2004 & $2001-2003$ & Maryland-Washington-Virginia & ILI & $\begin{array}{l}\text { Johns Hopkins APL ( } 300 \\
\text { drugstores in the Maryland- } \\
\text { Washington-Virginia area) }\end{array}$ & OTC & Day \\
\hline
\end{tabular}

\section{Proctor et al. [24] 1998}

993(Milkauwee)/1994 (Las egas)/1996 (Collingwood,

Collingwood (Ontario), Kelowna Vegas(Nevada)

\section{DRUG SELECTION STUDIES}

\section{Pelat et al. [33] 2010}

Cami et al. [31] 2009

2001-2003 
Table 1 General characteristics of studies included in a literature review of drug sales data analyses for surveillance of infectious diseases (Continued)

PREDICTION STUDIES

Vergu et al. [37] 2006

2000-2004

France

ILI

IMS Health (11,000 pharmacies,

OTC and prescribed

Week

Najmi et al. [36] 2005

2001-2003

Maryland-Washington-Virginia

ILI

$50 \%$ of all pharmacies in France)

Johns Hopkins APL (300

drugstores in the Maryland-

OTC

Day

Najmi et al. [35] 2004

2001-2002

Maryland-Washington-Virginia

ILI

Johns Hopkins APL (300

drugstores in the Maryland-

Abbreviations: ILI Influenza-like illness, GI Gastrointestinal, OTC Over-the-counter drugs. 
Table 2 Methodology and results of the retrospective studies included in a literature review of drug sales data analyses for surveillance of infectious diseases

\begin{tabular}{|c|c|c|c|c|c|c|c|c|c|}
\hline Author & Syndrome & $\begin{array}{l}\text { Reference data } \\
\text { sources }\end{array}$ & Drugs selected & $\begin{array}{l}\text { Statistical } \\
\text { methods }\end{array}$ & Correlation strength & $\begin{array}{l}\text { Correlation } \\
\text { timeliness }\end{array}$ & $\begin{array}{l}\text { Detection } \\
\text { sensitivity }\end{array}$ & $\begin{array}{l}\text { Detection } \\
\text { specificity }\end{array}$ & $\begin{array}{l}\text { Detection } \\
\text { timeliness }\end{array}$ \\
\hline \multicolumn{10}{|c|}{ STUDIES ON PRESCRIBED DRUGS } \\
\hline $\begin{array}{l}\text { Sugawara et al. } \\
\text { [27] } 2012\end{array}$ & $\mathrm{ILI}$ & $\begin{array}{l}\text { Influenza cases from } \\
5000 \text { hospitals and } \\
\text { clinics }\end{array}$ & $\begin{array}{l}\text { Drugs against } \\
\text { influenza virus : } \\
\text { oseltamivir, } \\
\text { zanamivir, } \\
\text { laninamivir }\end{array}$ & Correlation & $\begin{array}{l}\text { Pearson corr. coeff. } \\
r=0.992 \text { for } 2009 / 10 \text { and } \\
r=0.972 \text { for } 2010 / 11 \\
(p<0.001)\end{array}$ & - & - & - & - \\
\hline $\begin{array}{l}\text { Polgreen et al. } \\
\text { [23] } 2011\end{array}$ & $\mathrm{Gl}$ & $\begin{array}{l}\text { Hospitalizations with } \\
\text { diagnosis of } \\
\text { Clostridium Difficile } \\
\text { Infections }\end{array}$ & Oral vancomycin & $\begin{array}{l}\text { Cross-correlation, } \\
\text { Regression model }\end{array}$ & $\begin{array}{l}\text { Increase in Clostridium } \\
\text { Difficile Infections } \\
\text { associated with increase } \\
\text { in vancomycin use }\end{array}$ & - & - & - & - \\
\hline $\begin{array}{l}\text { Chopra et al. } \\
\text { [12] } 2011\end{array}$ & $\mathrm{ILI}$ & $\begin{array}{l}\text { Cases of influenza } \\
\text { reported from nine } \\
\text { sentinel healthcare } \\
\text { providers }\end{array}$ & Oseltamivir & Correlation & $\begin{array}{l}\text { Spearman corr.coeff. } \\
r=0.46(p<0.003) \text { Peaks } \\
\text { occurred at the same } \\
\text { time }\end{array}$ & - & - & - & - \\
\hline $\begin{array}{l}\text { Yoshida et al. } \\
\text { [30] } 2009\end{array}$ & $\mathrm{ILI}$ & $\begin{array}{l}28 \text { sentinel } \\
\text { surveillance sites of } \\
\text { influenza in Sahai City } \\
\text { (clinics and hospitals) }\end{array}$ & $\begin{array}{l}\text { Oseltamivir and } \\
\text { Zanamivir }\end{array}$ & Correlation & $\begin{array}{l}\text { Pearson corr.coeff. } \\
r=0.954\end{array}$ & - & - & - & - \\
\hline $\begin{array}{l}\text { Van den } \\
\text { Wijngaard et al. } \\
\text { [28] } 2008\end{array}$ & $\mathrm{ILI}$ & $\begin{array}{l}\text { Respiratory pathogen } \\
\text { diagnosis in } \\
\text { laboratory registries } \\
\text { (Influenza A, B, RSV, } \\
\text { enterovirus, S. } \\
\text { pneumoniae..) }\end{array}$ & $\begin{array}{l}\text { Drugs for } \\
\text { respiratory } \\
\text { infectious diseases } \\
\text { (7 ATC classes) }\end{array}$ & $\begin{array}{l}\text { Graphical } \\
\text { comparison, } \\
\text { Correlation, Linear } \\
\text { regression model }\end{array}$ & $\begin{array}{l}\text { Pearson corr .coeff. } \\
r=0.60 \text { for Influenza A, } r \\
=0.58 \text { for RSV, } r=0.60 \text { for } \\
\text { S. pneumonia, } r=0.39 \text { for } \\
\text { influenza B ( } p<0.05 \text { ) } 80 \% \\
\text { of variation explained by } \\
\text { respiratory pathogens }\end{array}$ & $\begin{array}{l}2 \text { weeks earlier } \\
\text { until } 1 \text { week later }\end{array}$ & - & - & - \\
\hline Author & Syndrome & $\begin{array}{l}\text { Reference data } \\
\text { sources }\end{array}$ & Drugs selected & $\begin{array}{l}\text { Statistical } \\
\text { methods }\end{array}$ & Correlation strength & $\begin{array}{l}\text { Correlation } \\
\text { timeliness }\end{array}$ & $\begin{array}{l}\text { Detection } \\
\text { sensitivity }\end{array}$ & $\begin{array}{l}\text { Detection } \\
\text { specificity }\end{array}$ & $\begin{array}{l}\text { Detection } \\
\text { timeliness }\end{array}$ \\
\hline $\begin{array}{l}\text { Chen et al. } \\
\text { [11] } 2005\end{array}$ & Pertussis & $\begin{array}{l}\text { Reported cases of } \\
\text { pertussis from the } \\
\text { NYS department of } \\
\text { Health }\end{array}$ & $\begin{array}{l}\text { Macrolide } \\
\text { antibiotics }\end{array}$ & CUSUM & - & - & $\begin{array}{l}100 \% \text { The signal } \\
\text { was indicator of } \\
\text { pertussis outbreak }\end{array}$ & $100 \%$ & $\begin{array}{l}\text { Not early } \\
\text { warning }\end{array}$ \\
\hline $\begin{array}{l}\text { Couturier et al. } \\
\text { [13] } 2004\end{array}$ & Syphilis & $\begin{array}{l}\text { Reported cases of } \\
\text { syphilis from } \\
\text { hospitals, physicians, } \\
\text { sexually transmitted } \\
\text { disease clinics. }\end{array}$ & $\begin{array}{l}\text { Benzylpenicillin } \\
\text { benzathine } 2.4 \\
\text { MUI }\end{array}$ & $\begin{array}{l}\text { Descriptive } \\
\text { analysis }\end{array}$ & $\begin{array}{l}\text { Similar trend }(+22 \% \\
\text { increase in Paris, }+10 \% \text { in } \\
\text { the } 5 \text { regions) }\end{array}$ & Similar trend & - & - & - \\
\hline \multicolumn{10}{|c|}{ STUDIES ON OTC DRUGS } \\
\hline $\begin{array}{l}\text { Kirian et al. } \\
\text { [19] } 2010\end{array}$ & Gl & $\begin{array}{l}\text { Cases of } \\
\text { gastrointestinal } \\
\text { diseases from County } \\
\text { Health Department } \\
\text { and detected Gl } \\
\text { outbreaks }\end{array}$ & $\begin{array}{l}\text { Diarrheal remedies } \\
\text { (based on } \\
\text { common use) }\end{array}$ & $\begin{array}{l}\text { Cross-correlation, } \\
\text { Regression ARIMA }\end{array}$ & $\begin{array}{l}\text { No significant correlation } \\
\text { between sales and Gl } \\
\text { cases counts, outbreak } \\
\text { counts. }\end{array}$ & - & $\begin{array}{l}\text { Not sensitive } 4 \%- \\
14 \%\end{array}$ & $\begin{array}{l}\text { Specific 97\%- } \\
100 \%\end{array}$ & - \\
\hline
\end{tabular}


Table 2 Methodology and results of the retrospective studies included in a literature review of drug sales data analyses for surveillance of infectious diseases (Continued)

\begin{tabular}{|c|c|c|c|c|c|c|c|c|c|}
\hline $\begin{array}{l}\text { Edge et al. } \\
\text { [17] } 2006\end{array}$ & $\mathrm{Gl}$ & $\begin{array}{l}\text { Counts of } \mathrm{Gl} \text { cases } \\
\text { due to bacteria, } \\
\text { parasites, and viruses }\end{array}$ & $\begin{array}{l}\text { Antinauseant and } \\
\text { antidiarrheal } \\
\text { products }\end{array}$ & Cross-correlation & $\begin{array}{l}\text { Temporal patterns of OTC } \\
\text { and Norovirus activity } \\
\text { were similar Pearson } \\
r^{2}=0.44\end{array}$ & No delay & - & - & - \\
\hline $\begin{array}{l}\text { Okhusa et al. } \\
\text { [22] } 2005\end{array}$ & ILI & $\begin{array}{l}\text { Reporting of patients } \\
\text { with ILI (hospitals, } \\
\text { clinics, physicians) }\end{array}$ & $\begin{array}{l}\text { Most common } \\
\text { treatments ILI }\end{array}$ & $\begin{array}{l}\text { Cross-correlation, } \\
\text { Prediction model, } \\
\text { Peak comparison }\end{array}$ & $\begin{array}{l}\text { Significant correlation } \\
\text { between sales and } \\
\text { influenza activity }\end{array}$ & $\begin{array}{l}\text { Sales do not } \\
\text { determine } \\
\text { influenza in } \\
\text { advance }\end{array}$ & - & - & - \\
\hline $\begin{array}{l}\text { Das et al. } \\
\text { [14] } 2005\end{array}$ & ILI & $\begin{array}{l}\text { Emergency } \\
\text { department in New } \\
\text { York City (ratio of ILI } \\
\text { syndrome visits/other } \\
\text { syndrome visits) }\end{array}$ & $\begin{array}{l}\text { A cold medication } \\
\text { selected } \\
\text { statistically from a } \\
\text { group of } 400 \text { cold } \\
\text { medications (ratio } \\
\text { ILI/analgesics } \\
\text { drugs sales) }\end{array}$ & $\begin{array}{l}\text { Cross-correlation, } \\
\text { Serfling method, } \\
\text { Graphical } \\
\text { comparison }\end{array}$ & $\begin{array}{l}\text { High correlation Pearson } \\
r^{2}=0.60(p<0.001)\end{array}$ & No lead time & $\begin{array}{l}\text { Sensitive (data not } \\
\text { reported) }\end{array}$ & $\begin{array}{l}\text { Not specific } \\
\text { (not reported) }\end{array}$ & $\begin{array}{l}\text { Not earlier } \\
\text { warning than } \\
\text { reference data }\end{array}$ \\
\hline $\begin{array}{l}\text { Das et al. } \\
\text { [14] } 2005\end{array}$ & $\mathrm{Gl}$ & $\begin{array}{l}\text { Emergency } \\
\text { department in New } \\
\text { York City (ratio of Gl } \\
\text { syndrome visits/other } \\
\text { syndrome visits) }\end{array}$ & $\begin{array}{l}\text { Common } \\
\text { antidiarrheal drugs } \\
\text { (ratio Gl/analgesic } \\
\text { drug sales) }\end{array}$ & $\begin{array}{l}\text { Cross-correlation, } \\
\text { Graphical } \\
\text { comparison }\end{array}$ & $\begin{array}{l}\text { Low correlation Pearson } \\
r^{2}=0.24(p<0.005) \\
\text { Similar increases during } \\
\text { the fall (norovirus) and } \\
\text { influenza peak. Increase in } \\
\text { ED GI visits during late } \\
\text { winter (rotavirus), but no } \\
\text { increase in drug sales. }\end{array}$ & - & $\begin{array}{l}\text { Less sensitive than } \\
\text { ED system }\end{array}$ & - & - \\
\hline Author & Syndrome & $\begin{array}{l}\text { Reference data } \\
\text { sources }\end{array}$ & Drugs selected & $\begin{array}{l}\text { Statistical } \\
\text { methods }\end{array}$ & Correlation strength & $\begin{array}{l}\text { Correlation } \\
\text { timeliness }\end{array}$ & $\begin{array}{l}\text { Detection } \\
\text { sensitivity }\end{array}$ & $\begin{array}{l}\text { Detection } \\
\text { specificity }\end{array}$ & $\begin{array}{l}\text { Detection } \\
\text { timeliness }\end{array}$ \\
\hline $\begin{array}{l}\text { Edge et al. } \\
\text { [16] } 2004\end{array}$ & $\mathrm{Gl}$ & $\begin{array}{l}\text { Emergency room } \\
\text { visits for acute } \mathrm{Gl}, \\
\text { number of } \mathrm{Gl} \text { cases } \\
\text { from case series } \\
\text { investigations } \\
\text { (waterborne outbreak) }\end{array}$ & $\begin{array}{l}\text { Saskatchewan: } \\
\text { four commonly } \\
\text { used antidiarrheals } \\
\text { and antinauseants } \\
\text { Ontario: } 12 \\
\text { products } \\
\text { (antidiarrheal, } \\
\text { antinauseant, } \\
\text { rehydration } \\
\text { products) }\end{array}$ & $\begin{array}{l}\text { Graphical } \\
\text { comparison } \\
\text { (Ontario, } \\
\text { Saskatchewan), } \\
\text { CUSUM, moving } \\
\text { average (Ontario) }\end{array}$ & $\begin{array}{l}\text { Trends of OTC products } \\
\text { comparable to the } \\
\text { outbreak epidemic curve } \\
\text { (Saskatchewan,Ontario) }\end{array}$ & - & $\begin{array}{l}100 \% \text { exceeded } \\
\text { threshold during } \\
\text { the outbreak } \\
\text { period (Ontario) }\end{array}$ & $100 \%$ & Not earlier \\
\hline \multirow[t]{2}{*}{$\begin{array}{l}\text { Magruder et al. } \\
\text { [21] } 2004\end{array}$} & ILI & $\begin{array}{l}\text { Outpatient insurance- } \\
\text { claim diagnoses for } \\
\text { acute respiratory con- } \\
\text { ditions, from 13,000 } \\
\text { clinics and doctors' } \\
\text { offices }\end{array}$ & $\begin{array}{l}\text { Remedies for } \\
\text { treating influenza } \\
\text { (common use) }\end{array}$ & Cross-correlation & $\begin{array}{l}\text { Seasonal trend: Pearson r } \\
\text { (between } 0.95 \text { and 0.99) }\end{array}$ & 1- 3 week lead & - & - & - \\
\hline & & & & & $\begin{array}{l}\text { Non-seasonal trend: } \\
\text { Pearson r (between } 0.25 \\
\text { and 0.75) }\end{array}$ & $\begin{array}{l}\text { No repeatable } \\
\text { lead time }\end{array}$ & & & \\
\hline
\end{tabular}


Table 2 Methodology and results of the retrospective studies included in a literature review of drug sales data analyses for surveillance of infectious diseases (Continued)

\begin{tabular}{|c|c|c|c|c|c|c|c|c|c|}
\hline $\begin{array}{l}\text { Hogan et al. } \\
\text { [18] } 2003\end{array}$ & ILI and Gl & $\begin{array}{l}\text { Hospital-discharge } \\
\text { diagnoses of } \\
\text { respiratory and } \\
\text { diarrheal disease in } \\
\text { children (for all } \\
\text { hospitals in } \\
\text { Pennsylvania, in Utah, } \\
\text { and } 95 \% \text { of Indiana). }\end{array}$ & $\begin{array}{l}\text { Electrolyte } \\
\text { products }\end{array}$ & $\begin{array}{l}\text { Cross-correlation, } \\
\text { EWMA }\end{array}$ & $\begin{array}{l}\text { Pearson } r=0.90(95 \% \mathrm{Cl}, \\
0.87-0.93)\end{array}$ & $\begin{array}{l}\text { Sales preceded } \\
\text { diagnoses by } \\
1.7 \text { weeks }(95 \% \mathrm{Cl} \text {, } \\
0.50-2.9)\end{array}$ & $100 \%$ & $100 \%$ & $\begin{array}{l}\text { Electrolyte } \\
\text { sales preceded } \\
\text { detection from } \\
\text { diagnoses by } \\
\text { an average of } \\
2.4 \text { weeks } \\
(95 \% \mathrm{Cl}, 0.1- \\
4.8) \text { Detection } \\
\text { earlier in 12/18 } \\
\text { outbreaks }\end{array}$ \\
\hline $\begin{array}{l}\text { Magruder } \\
\text { [20] } 2003\end{array}$ & $\mathrm{ILI}$ & $\begin{array}{l}\text { Outpatient insurance- } \\
\text { claim diagnoses for } \\
\text { acute respiratory } \\
\text { conditions }\end{array}$ & $\begin{array}{l}\text { Cold remedies: } \\
622 \text { products } \\
\text { (then grouped in } \\
\text { categories by } \\
\text { an expert in } \\
\text { pharmaco- } \\
\text { epidemiology) }\end{array}$ & Cross-correlation & Pearson $r=0.9$ & $\begin{array}{l}\text { Mean lead times } \\
\text { of } 2.8 \text { days }\end{array}$ & - & - & - \\
\hline $\begin{array}{l}\text { Davies et al. } \\
\text { [15] } 2003\end{array}$ & $\mathrm{ILI}$ & $\begin{array}{l}\text { Emergency admission } \\
\text { data from } \\
\text { Nottingham City } \\
\text { Hospital NHS Trust. }\end{array}$ & $\begin{array}{l}\text { Cold and flu } \\
\text { remedies (cold, } \\
\text { cough, } \\
\text { decongestant, } \\
\text { throat } \\
\text { preparation) }\end{array}$ & $\begin{array}{l}\text { Correlation, Peak } \\
\text { comparison, } \\
\text { Threshold } \\
\text { detection method }\end{array}$ & $\begin{array}{l}\text { National and local sales } \\
\text { positively correlated with } \\
\text { admissions in } 98 / 99 \text { and } \\
99 / 00 \text {, not 00/01 }\end{array}$ & - & $\begin{array}{l}100 \% \text { (for local } \\
\text { sales) }\end{array}$ & $\begin{array}{l}100 \% \text { (for local } \\
\text { sales) }\end{array}$ & $\begin{array}{l}\text { Rate of local } \\
\text { sales exceed } \\
\text { threshold of } \\
1000 \text { units per } \\
\text { week } 2 \text { weeks } \\
\text { prior to peak } \\
\text { in emergency } \\
\text { admissions }\end{array}$ \\
\hline Author & Syndrome & $\begin{array}{l}\text { Reference data } \\
\text { sources }\end{array}$ & Drugs selected & $\begin{array}{l}\text { Statistical } \\
\text { methods }\end{array}$ & Correlation strength & $\begin{array}{l}\text { Correlation } \\
\text { timeliness }\end{array}$ & $\begin{array}{l}\text { Detection } \\
\text { sensitivity }\end{array}$ & $\begin{array}{l}\text { Detection } \\
\text { specificity }\end{array}$ & $\begin{array}{l}\text { Detection } \\
\text { timeliness }\end{array}$ \\
\hline $\begin{array}{l}\text { Stirling et al. } \\
\text { [26] } 2001\end{array}$ & $\mathrm{Gl}$ & $\begin{array}{l}\text { Telephone survey } \\
\text { from a sample of } \\
\text { households: number } \\
\text { of persons with } \\
\text { diarrheal symptoms } \\
\text { and/or with stool } \\
\text { specimen positive to } \\
\text { C. parvum oocysts. } \\
\text { (waterborne outbreak) }\end{array}$ & $\begin{array}{l}\text { Common } \\
\text { antidiarrheal } \\
\text { (determined by } \\
\text { each pharmacist) }\end{array}$ & $\begin{array}{l}\text { Descriptive } \\
\text { analysis }\end{array}$ & $\begin{array}{l}\text { A fivefold increase in } \\
\text { sales during the epidemic } \\
\text { period }\end{array}$ & - & - & - & - \\
\hline $\begin{array}{l}\text { Proctor et al. } \\
\text { [24] } 1998\end{array}$ & $\mathrm{Gl}$ & $\begin{array}{l}\text { Comparison with } \\
\text { eight sources } \\
\text { (laboratory confirmed } \\
\text { cases of } \\
\text { Cryptosporidium, } \\
\text { clinically defined } \\
\text { cases) (waterborne } \\
\text { outbreak) }\end{array}$ & $\begin{array}{l}\text { Antidiarrheal: } \\
\text { Imodium, Pepto } \\
\text { Bismol, } \\
\text { Kaopectate }\end{array}$ & $\begin{array}{l}\text { Descriptive } \\
\text { analysis }\end{array}$ & $\begin{array}{l}\text { Significant increase in } \\
\text { drug sales during } \\
\text { epidemic period }\end{array}$ & - & - & - & - \\
\hline
\end{tabular}


Table 2 Methodology and results of the retrospective studies included in a literature review of drug sales data analyses for surveillance of infectious diseases (Continued)

\begin{tabular}{|c|c|c|c|c|c|c|c|c|}
\hline $\begin{array}{l}\text { Rodman et al. } \\
\text { [25] } 1997\end{array}$ & $\overline{\mathrm{Gl}}$ & $\begin{array}{l}\text { Cases of } \\
\text { cryptosporidiosis } \\
\text { (5 waterborne } \\
\text { outbreaks) }\end{array}$ & $\begin{array}{l}\text { Antidiarrheal } \\
\text { drugs }\end{array}$ & $\begin{array}{l}\text { Descriptive } \\
\text { analysis }\end{array}$ & $\begin{array}{l}\text { Milkauwee: increased } 20 \\
\text { fold; Las Vegas: no data; } \\
\text { Collingwood: increased in } \\
2 \text { of } 3 \text { stores;Kelowna: } \\
\text { increased } 3 \text { fold; } \\
\text { Cranbrook: increased }\end{array}$ & - & - & - \\
\hline $\begin{array}{l}\text { Welliver et al. } \\
\text { [29] } 1979\end{array}$ & ILI & $\begin{array}{l}\text { Laboratory count of } \\
\text { influenza B }\end{array}$ & $\begin{array}{l}\text { Children's aspirin, } \\
\text { adult antipyretics, } \\
\text { cold remedies }\end{array}$ & $\begin{array}{l}\text { Determination of } \\
\text { the\% of sales } \\
\text { increase, peak } \\
\text { comparison }\end{array}$ & $\begin{array}{l}\text { Sales of cold remedies } \\
\text { averaged } 185 \% \text { above the } \\
\text { baseline value during the } \\
\text { peak influenza activity }\end{array}$ & - & - & - \\
\hline
\end{tabular}

Abbreviations: ILI Influenza-like illness, GI Gastrointestinal, RSV Respiratory syncytial virus, ATC Anatomical Therapeutic Chemical classification system, CUSUM Cumulative sum control chart, OTC Over-the-counter drugs, EWMA Exponentially Weighted Moving Average. 
and hospital diagnoses of respiratory and diarrheal illness was 0.90 (95\% CI, 0.87-0.93) when drug sales were assumed to precede clinical diagnosis data by 1.7 weeks. Outbreaks were detected with $100 \%$ sensitivity and specificity in 3 of 5 studies that analyzed signal detection $[15,16,18]$. Drug sales data provided an earlier outbreak signal in two of them $[15,18]$. In Davies et al. [15], the rate of cough/cold sales exceeded a threshold of 1000 units per week two weeks before the peak in emergency department admissions during three consecutive winters. In Hogan et al. [18], detection from electrolytes sales occurred an average 2,4 weeks earlier than detection from hospital diagnoses of respiratory and diarrheal diseases.

Six of the seven studies that focused on prescribed drugs found strong correlations $(r=0.46-0.99)$ with clinical reference data or a significant increase in drug sales, without lead time however. The other study [11] showed that the CUSUM signal generated for prescriptions for macrolide antibiotics was linked to a pertussis outbreak in a county of New York State.

No association was observed between the type of reference data and the time lags observed.

\section{Drug selection studies}

An important challenge for drug-sales-based surveillance is identifying relevant indicator drug groups to monitor diseases. Five retrieved articles addressed this question. Characteristics of the studies are described in Table 3.

Two studies [21,34] developed methods to find homogeneous groups of OTC products. The authors used unsupervised clustering algorithms for aggregating OTC products in groups sharing similar sales histories. For example, Magruder et al. [21] first assigned OTC products for respiratory diseases to subgroups qualitatively based on indication, dose form, and age group. A stepwise hierarchical clustering algorithm was then used to form categories sharing a similar sales history, leading to a set of 16 product categories.

In two studies [31,33], the authors developed procedures to identify the drugs correlating with disease incidence. Clusters were formed specifically for a particular disease. In Pelat et al. [33], a hierarchical clustering procedure was applied to the time series of all therapeutic classes and the acute diarrhea incidence rate reported by a network of general practitioners. Four therapeutic classes were found to cluster with diarrhea incidence and an algorithm based on the selected drugs allowed the detection of epidemics with a sensibility of $100 \%$, a specificity of $95 \%$ and a timeliness of 1.7 weeks before official alerts.

\section{Prediction studies}

In three studies [35-37], the authors developed models to predict clinical data based on drug sales data.

Vergu et al. [37] used a Poisson regression model on selected OTC sales to forecast influenza-like illness (ILI) incidence as recorded by a sentinel network of general practitioners. The forecast at the national level 1-3 weeks ahead showed a strong correlation with observed ILI incidence $(\mathrm{r}=0.85-0.96)$.

Najmi et al. [35] used least mean square filtering methods to estimate the incidence of emergency room consultations for respiratory conditions from past and present sales of groups of cold-remedy sales. In a later article [36], they succeeded in extending the estimation algorithm for predicting increases in clinical data several days in advance.

\section{Discussion}

The evidence gathered in this systematic literature review suggests that drug sales data analysis can be a useful tool

Table 3 Methodology and results of drug selection studies included in a literature review of drug sales data analysis for surveillance of infectious diseases

\begin{tabular}{|c|c|c|c|}
\hline Author & Disease & Method & Results of the algorithm evaluation \\
\hline Pelat et al. [33] 2010 & Gl & Hierarchical clustering procedure ,CUSUM & $\begin{array}{l}\text { Identification of } 4 \text { therapeutic classes relevant to gastroenteritis } \\
\text { outbreak detection. Detection performance of a multiple voter } \\
\text { algorithm: sensibility } 100 \% \text {, specificity } 95 \% \text {, timeliness } \\
1.7 \text { weeks. }\end{array}$ \\
\hline Cami et al. [31] 2009 & $\mathrm{ILI}$ & Aggregate mining algorithm & $\begin{array}{l}\text { Identification of product categories with outbreak detection } \\
\text { performance superior to predefined categories and more } \\
\text { strongly correlated with the disease data. }\end{array}$ \\
\hline Wallstrom et al. [34] 2007 & $\mathrm{ILI}$ & Unsupervised time-series clustering algorithm & $\begin{array}{l}\text { Distinction between OTC products for allergy and OTC } \\
\text { products for influenza symptoms }\end{array}$ \\
\hline Li et al. [32] 2005 & $|L| / G \mid$ & Canonical correlation analysis & $\begin{array}{l}\text { Identification of eight diagnoses that have strong association } \\
\text { with electrolyte sales }(r=0.96)\end{array}$ \\
\hline Magruder et al. [21] 2004 & $\mathrm{ILI}$ & Unsupervised stepwise clustering algorithm & $\begin{array}{l}\text { Identification of } 16 \text { OTC product groups with similar historical } \\
\text { trends }\end{array}$ \\
\hline
\end{tabular}


for surveillance of acute respiratory and gastrointestinal infections.

As could be expected, prescribed drug sales data were strongly correlated with clinical case reporting. No lead time was observed, which is consistent with the fact that patients purchase drugs after seeing a healthcare professional. Analysis of prescribed drug sales data may nevertheless have an additional utility for epidemic detection, as these data might be available with a shorter delay than clinical surveillance data [27].

A high correlation between OTC drug sales data and reference surveillance data were found in almost all the retrospective studies. Several studies also showed that OTC drug sales can serve as an early indicator of disease epidemics. Patients may buy nonprescription drugs during the early phase of illness when they become symptomatic, before consulting a health practitioner [38]. A surveillance system based on drug data should ideally detect all the outbreaks, rapidly, with a low false alert rate. However, few studies in the review determined the sensitivity and specificity of the outbreak detection and those aspects should be analyzed in more details in future studies.

Surveillance based on OTC drug sales could be particularly relevant for diseases whose prodromal phase persists for several days before the onset of more severe symptoms. For example, the early stages of dengue fever symptoms are nonspecific (fever, headache, myalgia, arthralgia, etc.) [39]. The occurrence of grouped cases could trigger an excess of nonspecific drug sales over baseline levels, which in turn could provide an early warning of outbreak in an endemic area.

Results from drug selection studies showed that it is possible to identify groups of products strongly associated with incidence data, which can then be used to predict future trends in clinical data and help public health authorities to prepare response planning. Such product selection procedures, however, depend on the existence of large clinical surveillance databases of the diseases concerned.

Similarly, the validity of drug sales data analysis has been evaluated mainly for two disease groups, respiratory and gastrointestinal illness, for which clinical reference data, used as the gold standard, are readily available. Pertussis and syphilis have been evaluated in only one study each, and still require further confirmation. The concept of drug-based surveillance therefore needs to be validated for other infectious diseases.

All the studies were conducted in developed countries or area. Surveillance based on drug sales data requires electronic information systems for routine data analysis. Besides, its implementation requires that the population has access to the health care system and mainly buy drugs in pharmacies. This could limit the use of drug based surveillance systems in developing countries.
By improving the timeliness of epidemic detection compared to clinical data and giving information from a larger part of the population, drug sales data can be an additional source of information for already monitored diseases. Besides, drug sales data analysis could have its greatest value in the surveillance of diseases for which clinical surveillance is cumbersome and costly, or where substantial under-reporting is suspected. To confirm the selected drug group as a valid proxy of disease, clinical surveillance may be conducted for a defined period in a representative population. Examples of diseases for which this would be useful are typically varicella, urinary infections, allergies/asthma, and parasitic diseases.

Ideally, the drugs to be monitored should be specific to the disease and widely used to treat it in order to maximize the sensitivity of the signal. For example, benzylpenicillin benzathine 2.4 MUI is the quasi exclusive treatment for syphilis infection [13] and is a good candidate. In contrast, the treatment of measles is mostly symptomatic without a specific drug, which makes this disease unattractive for this approach. Another limitation applies to diseases that are usually treated in hospitals or specialized centers, such as tuberculosis.

Surveillance based on drug sales, may not be appropriate to accurately estimate incidence of diseases, as the source population size is not precisely known. Moreover, it may be difficult to link the number of drug packages sold to the number of patients with disease. However, the method is very efficient to determine temporal dynamics of a situation and to detect abnormal phenomena. Surveillance based on drug sales is therefore well adapted to diseases with seasonal variations such as norovirus gastroenteritis, influenza and other infectious respiratory agents, or community outbreaks (foodborne illnesses, waterborne illnesses, hepatitis A, etc.).

Drug sales can be influenced by store promotions, sales period (holidays, weekends), and the media. Also, we do not know whether people buy medications to treat a disease they currently have or a disease they fear they may have in the near future. For example, during the media coverage of avian influenza A (H5N1) in the US, an increase in antiviral medications sales was observed [40], which corresponded to stockpiling behavior of the population.

Health-seeking behaviors also vary by demographic, social, cultural, and economic factors. A survey [41] in Canada analyzed the healthcare-seeking behaviors of 351 patients with acute gastroenteritis. They found significant differences (patient age and sex) between the patients who used OTC drugs and those who did not. Consequently, factors that prompt self-medication should also be taken into account. The usefulness of drug sales based surveillance is also dependent on the available resources and the organization of the health care system. OTC drug sales 
surveillance is for example less relevant in countries where reimbursement rate are high and patients mainly get prescribed drugs.

Population mobility, particularly in tourist areas, may lead to an increase in remedy sales, which could wrongly be interpreted as a disease outbreak. Inversely, patients with high geographical mobility may not be included in the region of study and lead to an underestimation of the magnitude of an epidemic.

Despite some limitations, routine collection and analysis of drug sales data are likely to be developed in the coming years. Many automated surveillance systems that collect drug data the day after the sales have been implemented in the last decade $[14,19,20,27]$. They allow a rapid assessment of the public health situation. Early detection of outbreaks allows public health authorities to set up epidemic investigations and control measures sooner. Most studies included in this review were published after the year 2000, with their number increasing recently. They illustrate the need for improved surveillance systems, evidenced by recent public health crises (e.g., anthrax in 2001, the SARS outbreak in 2003, the A/H1N1 influenza pandemic in 2009 , etc.). Drug sales data present indeed many advantages in terms of public health surveillance. Data can be obtained in a real-time manner and usually cover a large portion of the population. Data collection may be exhaustive, without selection of specific sales, and allows the simultaneous monitoring of a large number of diseases, especially new or emerging diseases.

Although non-specific, drug sales data are directly linked to patients' health conditions. Drug sales data are therefore more specific than other syndromic surveillance data, such as tracking search patterns on the web and are likely to reflect more accurately disease activity in the population. Moreover, it should be noted that alternative sources of data for disease surveillance are currently under development. Healthcare management databases that can provide exhaustive information on drug consumption and diagnosis, as the Dossier Médical Personnel [42] in France, are promising tools for disease surveillance.

Our review may be affected by a publication bias since studies unable to show correlations between drug sales and reference data may have been less published. In addition, selections bias may have occurred in the studies. Indeed, some studies in the review were based on a limited number of pharmacies and/or a limited study period (e.g. less than one year). Language bias may exist as we were not able to identify studies published in languages other than English and French. The review focused on the temporal dynamics of infectious disease; consequently, further analyses are required to determine the capacity of these systems to efficiently monitor other aspects of infectious diseases such as spatial spreading.

\section{Conclusion}

This review suggests that the analysis of drug sales data is a promising method for surveillance and outbreak detection of infectious diseases. It has the potential to trigger an outbreak alert earlier than most surveillance systems. However, the main challenges consist in the appropriate selection of indicator drug groups and the validation of this approach for diseases for which no or poor quality clinical surveillance data exists. The usefulness of the approach also depends on the available resources and the organization of the health care system. Drug sales databases with real-time or near real-time data transmission are available in several countries; future studies should be encouraged to expand their use on other infectious diseases.

\section{Competing interests}

Mathilde Pivette prepares a doctoral thesis under the French framework "CIFRE" (Industrial contract for training through research; www.anrt.asso.fr), in partnership with the company Celtipharm (www.celtipharm.org). The other authors declare they have no competing interests.

\section{Authors' contributions}

All authors contributed to the study's design. MP and JM carried out the literature search and reviewed articles. MP drafted the manuscript. All authors interpreted the results, revised and approved the final manuscript.

\section{Authors' information}

Mathilde Pivette has a Doctor of Pharmacy degree and a Master of Public Health. She is a PhD candidate in epidemiology at the French School of Public Health. Her research interest lies in the analysis of drug sales data for disease surveillance.

\section{Acknowledgements}

This research was funded by Celtipharm (Vannes, France) a company specialized in the real time collection and statistical processing of healthcare data (www.celtipharm.org - www.openhealth.fr), through a doctoral thesis contract for Mathilde Pivette.

\section{Author details}

${ }^{1}$ EHESP French School of Public Health, Sorbonne Paris Cité, Rennes, France. ${ }^{2}$ Université Paris Descartes, MAP5, Paris, France. ${ }^{3}$ Celtipharm, Vannes, France. ${ }^{4}$ Institut Pasteur, Emerging Diseases Epidemiology Unit, Paris, France. ${ }^{5}$ Aix Marseille Université, IRD French Institute of Research for Development, EHESP French School of Public Health, UMR_D 190 "Emergence des Pathologies Virales", Marseille, France.

Received: 24 May 2013 Accepted: 31 October 2014

Published online: 18 November 2014

\section{References}

1. Henning KJ: What is syndromic surveillance? MMWR Morb Mortal Wkly Rep 2004, 53(Suppl):5-11

2. CDC: Framework for evaluating public health surveillance systems for early detection of outbreaks: recommendations from the CEC working group. MMWR Morb Mortal Wkly Rep 2004, 53(RR-5):1-11.

3. Berger M, Shiau R, Weintraub JM: Review of syndromic surveillance: implications for waterborne disease detection. J Epidemiol Community Health 2006, 60:543-550.

4. Kara EO, Elliot AJ, Bagnall H, Foord DGF, Pnaiser R, Osman H, Smith GE, Olowokure B: Absenteeism in schools during the 2009 influenza $A(H 1 N 1)$ pandemic: a useful tool for early detection of influenza activity in the community? Epidemiol Infect 2011, 140:1-9.

5. Rolland E, Moore KM, Robinson VA, McGuinness D: Using Ontario's "Telehealth" health telephone helpline as an early-warning system: a study protocol. BMC Health Serv Res 2006, 6:10. 
6. Rodman JS, Frost F, Jakubowski W: Using nurse hot line calls for disease surveillance. Emerg Infect Dis 1998, 4:329-332.

7. Lewis MD, Pavlin JA, Mansfield JL, O'Brien S, Boomsma LG, Elbert Y, Kelley PW: Disease outbreak detection system using syndromic data in the greater Washington DC area. Am J Prev Med 2002, 23:180-186.

8. Josseran L, Fouillet A, Caillère N, Brun-Ney D, llef D, Brucker G, Medeiros H, Astagneau P: Assessment of a syndromic surveillance system based on morbidity data: results from the Oscour network during a heat wave. PLoS One 2010, 5:e11984.

9. Mostashari F, Fine A, Das D, Adams J, Layton M: Use of ambulance dispatch data as an early warning system for communitywide influenzalike illness, New York City. J Urban Heal 2003, 80(2 Suppl 1):i43-i49.

10. Moher D, Liberati A, Tetzlaff J, Altman DG: Preferred reporting items for systematic reviews and meta-analyses: the PRISMA statement. BMJ 2009, 339:b2535.

11. Chen J-H, Schmit K, Chang H, Herlihy E, Miller J, Smith P: Use of Medicaid prescription data for syndromic surveillance-New York. MMWR Morb Mortal Wkly Rep 2005, 54(Suppl):31-34.

12. Chopra T, Binienda J, Mohammed M, Shyamraj R, Long P, Bach D, Carlton C, Peters S, Lephart P, Alangaden G, Dhar S, Marchaim D, Schreiber M, Kaye KS: A practical method for surveillance of novel H1N1 influenza using automated hospital data. Infect Control Hosp Epidemiol 2011, 32:700-702.

13. Couturier E, Michel A, Janier M, Dupin N, Semaille C: Syphilis surveillance in France, 2000-2003. Euro Surveill 2004, 9:8-10.

14. Das D, Metzger K, Heffernan R, Balter S, Weiss D, Mostashari F: Monitoring over-the-counter medication sales for early detection of disease outbreaks-New York City. MMWR Morb Mortal Wkly Rep 2005, 54(Suppl):41-46.

15. Davies GR, Finch RG: Sales of over-the-counter remedies as an early warning system for winter bed crises. Clin Microbiol Infect 2003, 9:858-863.

16. Edge VL, Pollari F, Lim G, Aramini J, Sockett P, Martin SW, Wilson J, Ellis A: Syndromic surveillance of gastrointestinal illness using pharmacy over-the-counter sales. a retrospective study of waterborne outbreaks in Saskatchewan and Ontario. Can J Public Heal 2004, 95:446-450.

17. Edge $V L$, Pollari F, Ng LK, Michel P, McEwen S a, Wilson JB, Jerrett M Sockett PN, Martin SW: Syndromic surveillance of norovirus using over-the-counter sales of medications related to gastrointestinal illness. Can J Infect Dis Med Microbiol 2006, 17:235-241.

18. Hogan WR, Tsui F-C, Ivanov O, Gesteland P, Grannis S, Overhage JM: Detection of pediatric respiratory and diarrheal outbreaks from sales of over-the-counter electrolyte products. J Am Med Informatics Assoc 2003, 10:555-562.

19. Kirian ML, Weintraub JM: Prediction of gastrointestinal disease with over-the-counter diarrheal remedy sales records in the San Francisco Bay Area. BMC Med Inform Decis Mak 2010, 10:39.

20. Magruder SF: Evaluation of over-the-counter pharmaceutical sales as a possible early warning indicator of human disease. John Hopkins Univ Appl Phys Lab Tech Dig 2003, 24:349-353.

21. Magruder SF, Lewis SH, Najmi A, Florio E: Progress in understanding and using over-the-counter pharmaceuticals for syndromic surveillance. MMWR Morb Mortal Wkly Rep 2004, 53(Suppl):117-122.

22. Ohkusa Y, Shigematsu M, Taniguchi K, Okabe N: Experimental surveillance using data on sales of over-the-counter medications-Japan, November 2003-April 2004. MMWR Morb Mortal Wkly Rep 2005, 54 Suppl:47-52.

23. Polgreen PM, Yang M, Kuntz JL, Laxminarayan R, Cavanaugh JE: Using oral vancomycin prescriptions as a proxy measure for Clostridium difficile infections: a spatial and time series analysis. Infect Control Hosp Epidemiol 2011, 32:723-726.

24. Proctor ME, Blair KA, Davis JP: Surveillance data for waterborne illness detection: an assessment following a massive waterborne outbreak of Cryptosporidium infection. Epidemiol Infect 1998, 120:43-54.

25. Rodman JS, Frost F, Davis-Burchat L: Pharmaceutical sales; a method for disease surveillance? J Environ Health 1997, 60:8-14.

26. Stirling R, Aramini J, Ellis A, Lim G, Meyers R, Fleury M, Werker D: Waterborne cryptosporidiosis outbreak. Canada Commun Dis Rep 2001 27:185-192.

27. Sugawara T, Ohkusa Y, Ibuka Y, Kawanohara H, Taniguchi K, Okabe N: Real-time prescription surveillance and its application to monitoring seasonal influenza activity in Japan. J Med Internet Res 2012, 14:e14.

28. Van den Wijngaard C, van Asten L, van Pelt W, Nagelkerke NJD, Verheij R, de Neeling AJ, Dekkers A, van der Sande MAB, van Vliet H, Koopmans MPG:
Validation of syndromic surveillance for respiratory pathogen activity. Emerg Infect Dis 2008, 14:917-925.

29. Welliver RC, Cherry JD, Boyer KM, Deseda-Tous JE, Krause PJ, Dudley JP, Murray RA, Wingert W, Champion JG, Freeman G: Sales of nonprescription cold remedies: a unique method of influenza surveillance. Pediatr Res 1979, 13:1015-1017.

30. Yoshida M, Matsui T, Ohkusa Y, Kobayashi J, Ohyama T, Sugawara T, Yasui Y, Tachibana T, Okabe N: Seasonal influenza surveillance using prescription data for anti-influenza medications. Jpn J Infect Dis 2009, 62:233-235.

31. Cami A, Wallstrom GL, Fowlkes AL, Panozzo CA, Hogan WR: Mining aggregates of over-the-counter products for syndromic surveillance. Pattern Recognit Lett 2009, 30:255-266.

32. Li R, Wallstrom GL, Hogan WR: A multivariate procedure for identifying correlations between diagnoses and over-the-counter products from historical datasets. In AMIA Annu Symp Proc. 2005:450-454.

33. Pelat C, Boëlle P-Y, Turbelin C, Lambert B, Valleron A-J: A method for selecting and monitoring medication sales for surveillance of gastroenteritis. Pharmacoepidemiol Drug Saf 2010, 19:1009-1018.

34. Wallstrom GL, Hogan WR: Unsupervised clustering of over-the-counter healthcare products into product categories. J Biomed Inform 2007, 40:642-648

35. Najmi A-H, Magruder SF: Estimation of hospital emergency room data using otc pharmaceutical sales and least mean square filters. BMC Med Inform Decis Mak 2004, 5:1-5.

36. Najmi A-H, Magruder SF: An adaptive prediction and detection algorithm for multistream syndromic surveillance. BMC Med Inform Decis Mak 2005, 5:33.

37. Vergu E, Grais RF, Sarter H, Fagot J-P, Lambert B, Valleron A-J, Flahault A Medication sales and syndromic surveillance, France. Emerg Infect Dis 2006, 12:416-421.

38. Mandl KD, Overhage JM, Wagner MM, Lober WB, Sebastiani P, Mostashari F, Pavlin J, Gesteland P, Treadwell T, Koski E, Hutwagner L, Buckeridge DL, Aller $\mathrm{RD}, \mathrm{Grannis} \mathrm{S:} \mathrm{Implementing} \mathrm{syndromic} \mathrm{surveillance} \mathrm{:} \mathrm{a} \mathrm{practical} \mathrm{guide}$ informed by the early experience. J Am Med Informatics Assoc 2004, 11:141-150.

39. Meynard J-B, Chaudet H, Texier G, Ardillon V, Ravachol F, Deparis X, Jefferson $H$, Dussart $P$, Morvan J, Boutin J-P: Value of syndromic surveillance within the Armed Forces for early warning during a dengue fever outbreak in French Guiana in 2006. BMC Med Inform Decis Mak 2008, 8:29.

40. Miller J, Schmit K, Duncan P, Waters C: Increased antiviral medication sales before the 2005-06 influenza season-New York City. MMWR Morb Mortal Wkly Rep 2006, 55:277-279.

41. Frosst GO, Majowicz SE, Edge VL: Factors associated with the use of over-the-counter medications in cases of acute gastroenteritis in Hamilton, Ontario. Can J public Heal 2006, 97:489-493.

42. DMP- Dossier Médical Personnel. www.dmp.gouv.fr.

doi:10.1186/s12879-014-0604-2

Cite this article as: Pivette et al: Drug sales data analysis for outbreak detection of infectious diseases: a systematic literature review. BMC Infectious Diseases 2014 14:604.

\section{Submit your next manuscript to BioMed Central and take full advantage of:}

- Convenient online submission

- Thorough peer review

- No space constraints or color figure charges

- Immediate publication on acceptance

- Inclusion in PubMed, CAS, Scopus and Google Scholar

- Research which is freely available for redistribution 